\title{
ATTACHMENT RETAINED OVERDENTURE
}

\author{
Sheen J. Arora1., Aman Arora ${ }^{2}$., Ritu Sangwan ${ }^{3}$ and Prachi Jain ${ }^{4 *}$ \\ 1,2,3,4 Department of Prosthodontics, Room no. 5, J.N. Kapoor, D.A.V.C Dental College, \\ Model Town, Yamunanagar, Haryana 135001 \\ ${ }^{3}$ Private practioner, Rohtak
}

DOI: http://dx.doi.org/10.24327/ijrsr.2017.0803.0094

\section{ARTICLE INFO}

\section{Article History:}

Received $15^{\text {th }}$ December, 2016

Received in revised form $25^{\text {th }}$

January, 2017

Accepted $23^{\text {rd }}$ February, 2017

Published online $28^{\text {th }}$ March, 2017

\begin{abstract}
The goal of prosthetic dentistry is to restore patient's masticatory function and aesthetics along with preservation of the remaining intraoral structures. This can be achieved by preventive prosthodontics which is in compliance with Devan's dictum stating that "preservation of the remaining structure is more important than the replacement of what is missing". Preventive prosthodontics helps to improve retention and stability of the final prosthesis. Moreover, it preserves the alveolar bone and proprioception, providing psychological benefit to the patient, thereby enhancing the success rate of the treatment. This article presents a case report of rehabilitation of a partially edentulous patient incorporating the principles of preventive prosthodontics.
\end{abstract}

Copyright @ Sheen J. Aror et al, 2017, this is an open-access article distributed under the terms of the Creative Commons Attribution License, which permits unrestricted use, distribution and reproduction in any medium, provided the original work is properly cited.

\section{INTRODUCTION}

Since several years, the most widely used treatment modality in patients reporting with multiple missing teeth or having mutilated teeth or badly broken teeth or teeth having severe periodontal involvement, has been extraction of all the teeth followed by complete denture (Tiwari P et al, 2013). But this treatment leads to poor masticatory efficiency and loss of neuromuscular function due to decrease in the proprioceptive response resulting from loss of teeth (Tiwari $\mathrm{P}$ et al 2013, Winkler S 2009). Also, this results in accelerated rate of bone resorption. Moreover, the poor retention of the prosthesis leads to poor acceptance by the patient adding to the psychological dissatisfaction.

With the recent introduction of implants, the disadvantages of removable denture have been overcome but it has its own demerits as implant treatment cannot be implemented in every case. Various systemic factors contraindicate implant treatment such as recent myocardial infarction, uncontrolled diabetes, severe renal disease, valvular prosthesis and untreated osteomalacia and osteoporosis etc. The local factors which need to be assessed include the height and width of the available bone, periodontal biotype and the general factors include the age, patient's compliance and cooperation. Also, the long waiting period and increased cost of the implant treatment is not acceptable to every patient and the different loading protocols available make the procedure technique sensitive. Thus, implant treatment can be implemented in only limited number of patients.

Attachment retained overlay denture in this context is the treatment of choice in such patients. It is also in compliance with the Devan's dictum. It is defined as any removable dental prosthesis that covers and rests on one or more remaining natural teeth, the roots of natural teeth and/or dental implants (GPT-8, 2005). Though it is a removable prosthesis, but as it preserves natural teeth or roots, it is better than other treatment options. By preserving natural teeth, proprioception is maintained along with preservation of the alveolar bone which results in increased retention, stability, support and the masticatory efficiency.

This case report presents tooth-supported overlay denture with castable bar attached to the lower denture with an indirect technique. The design incorporates use of plastic retention clips inside a metal superstructure. This gives the added advantage of plastic clip removal and replacement with new clip after wear or loosening of the existing clip.

\section{CASE REPORT}

A fifty six years old male patient reported to our department with the chief complaint of poor aesthetics and difficulty in chewing food due to missing teeth. He wanted to get his missing teeth replaced. There was no relevant medical history affecting prosthodontic treatment. Extra oral examination showed no gross abnormality (Figure 1). 


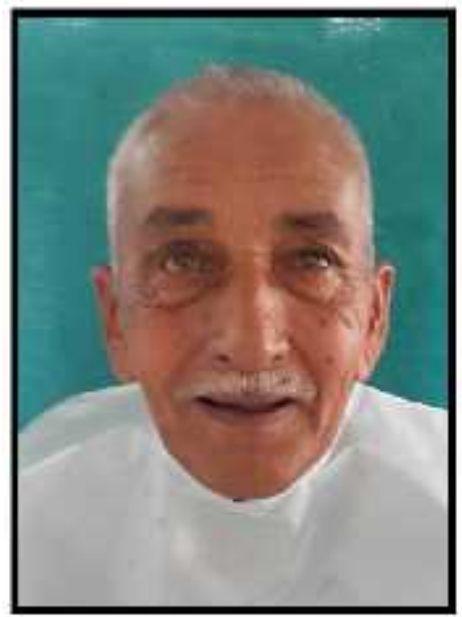

Fig 1 Pre-Rehabilitation extraoral view

The clinical and radiographic examination revealed the edentulous maxillary arch and 33, 34, 37, 43, 44 \& 47 teeth present in mandibular arch, with no periapical pathology. The teeth were periodontally sound, with no mobility and patient exhibited class I ridge relationship with sufficient interarch space.

\section{Procedure}

\section{Primary impression and preparation of the abutment}

Preliminary maxillary and mandibular impression were made with irreversible hydrocolloid impression material and preliminary cast were obtained. Intentional root canal treatment was performed on canines, premolars and second molars of the mandibular arch (Figure 2) after which they were prepared with a tapered round end diamond rotary bur with a chamfer finish line for the metal copings. The preparation was rounded to minimize the horizontal torque on the roots. They were reduced in vertical height to $2 \mathrm{~mm}$ above the crest of the ridge to provide sufficient space for the overdenture prosthesis.

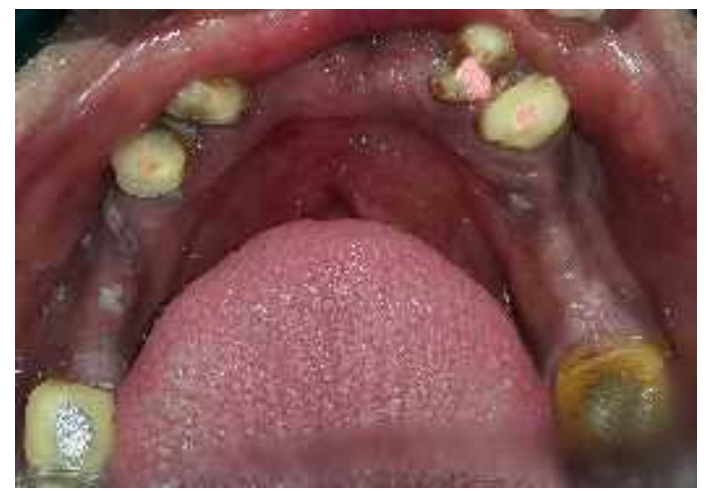

Fig. 2 Intraoral view of mandibular arch after intentional root canal treatment on remaining teeth

\section{Final impression}

After the preparation of the abutments, mandibular impression was made in the custom tray fabricated on the primary cast with polyvinyl siloxane elastomeric impression material (putty and light body) using double step putty wash technique and cast was poured using die stone. Simultaneously, maxillary border moulding was done and secondary impression was made using zinc oxide eugenol impression paste. This impression was poured in dental stone and maxillary master cast was obtained.

\section{Tentative maxilla-mandibular relation}

Trial denture bases were fabricated over these casts obtained and occlusion rims were fabricated over the bases after which a tentative maxillomandibular relation was recorded to determine the space available for the Hader bar.

\section{Fabrication of copings}

Inlay wax patterns of copings were fabricated for the prepared canines, premolars and second molars. The two wax copings on the canines were connected with a pre-fabricated plastic bar (Preci-clix: Figure 3) of $2 \mathrm{~mm}$ thickness and $3 \mathrm{~mm}$ height.

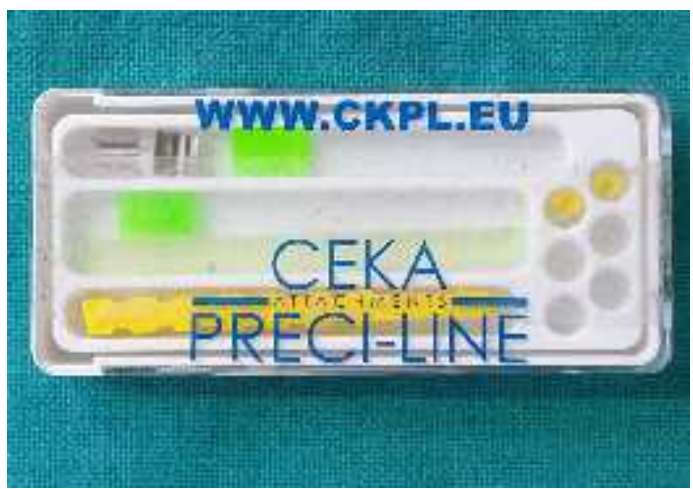

Fig. 3 Prefabricated Preci-clix plastic bar attachment

The wax pattern was cast in a Ni-Cr alloy using standard technique. Casting was than retrieved, finished and polished to avoid any plaque accumulation along the bar (Figure 4).

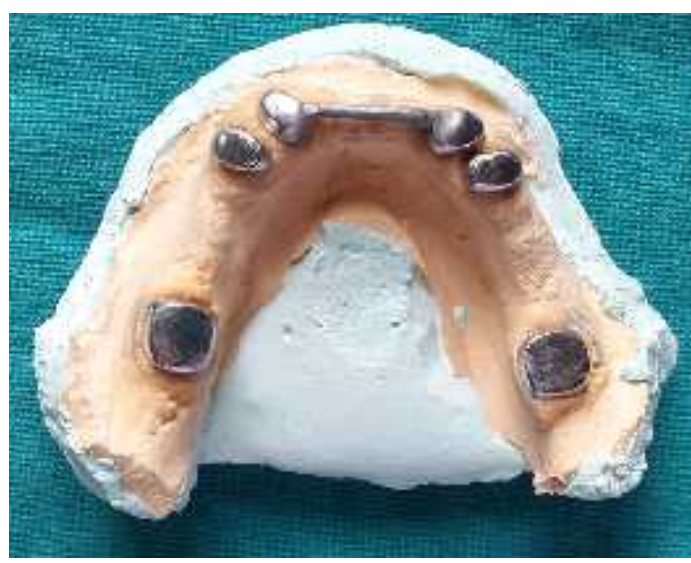

Fig. 4 Finished casting on the mandibular cast

\section{Cementation of the copings}

The metal bars with retainer copings were first tried intraorally to check for the passive fit. Thereafter, the metal copings with attached bar were luted onto the respective tooth preparations i.e. canines using glass ionomer cement. Similarly, the metal copings for premolars and molars were also luted using the same cement.

\section{Fabrication of metal framework and its try in}

Full wax spacer was adapted on the mandibular cast and custom tray was fabricated. After blocking the undersurface of the hader bar and placing a plastic positioned clip on the mid surface of the bar, final impression was made using putty and 
light body impression material and master cast was poured. This master cast was duplicated and a metal framework was fabricated over the duplicated cast and tried-in patient's mouth.

\section{Final jaw relation and trial of waxed complete denture}

Jaw relations were recorded and transferred to a semi adjustable articulator using face bow. The artificial teeth were selected and arranged on the record bases for a trial denture arrangement and they were evaluated intraorally for phonetics, aesthetics, occlusal vertical dimension and centric relation. A protrusive record was made, to set the articulator's condylar elements and to achieve a balanced occlusal arrangement.

\section{Denture processing and finishing}

After the trial and wax up, flasking and dewaxing was done after which the metal framework was placed on the duplicated master cast. The undersurface of the metal framework was blocked to avoid flow of resin between the positioned clip and bar.

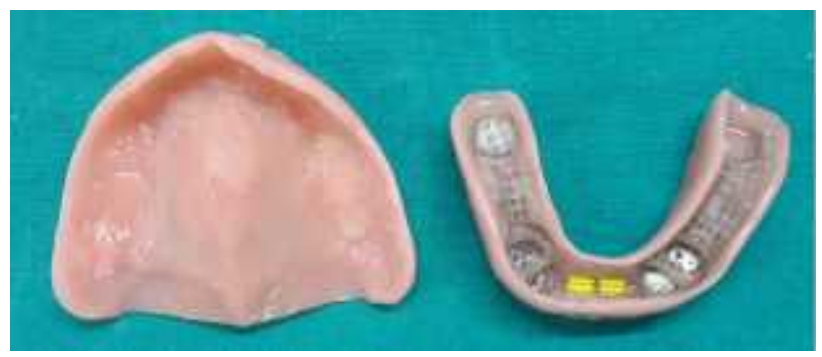

Fig. 5 Intaglio surface of the finished maxillary and mandibular prosthesis with retentive clip in mandibular prosthesis

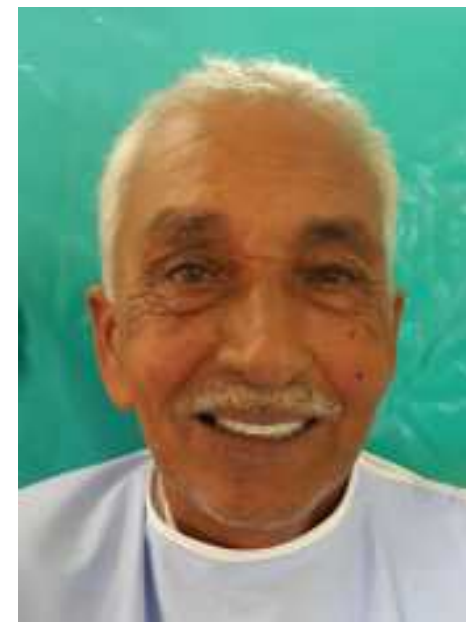

Fig. 6 Post rehabilitation extraoral view
The dentures were then processed in the conventional manner after which finishing was done and retentive clip was placed in place of the positioned clip (Figure 5). The denture was inserted and patient was trained for insertion and removal of the denture (Figure 6).

\section{DISCUSSION}

In this article, bar retained overlay denture has been described. This treatment modality was planned as the patient was not willing to undergo extraction of the remaining teeth. Thus "preventive prosthodontics," in the form of overlay denture therapy was applied. Overdenture was retained with bar attachment because bar is positioned close to the mandibular alveolar bone, torquing forces applied through the bar will be less than the torquing forces applied through the occlusal rest of a mandibular partial denture (Mishra $\mathrm{R}$ et al, 2014). Overdenture provided a stable base for the prosthesis, and enabled utilization of the advances in periodontal and endodontic therapy in conjunction with a greatly reduced crown-to root ratio to give a viable treatment to minimize ill effects of a conventional denture (Tiwari et al, 2013).

Rehabilitation of a partially edentulous patient with few remaining teeth by overlay denture has become a common treatment modality. This not only preserves the residual alveolar bone but also increases masticatory efficiency, preserves proprioception and increases support and retention of the prosthesis. The retention in overdentures is increased with the help of attachments which are attached to the abutment copings. Attachment retained overdentures helps in distribution of masticatory forces, minimizes trauma to abutments and soft tissues, attenuate ridge resorption, improves the esthetics and retains proprioception. Presence of teeth gives a psychological impact on the person's mind of having natural teeth in his mouth, which adds to the personal confidence.

\section{References}

1. Tiwari P, Karambelkar V, Patel J, Naveen Y.G., Javiya P, Sethuraman R. Attachment retained overdenture. Eur J Dent Therapy Res 2013; 3:167-169.

2. Winkler S. Essentials of complete denture Prosthodontics. India: AITBS Publishers, 2009.

3. The glossary of prosthodontic terms (GPT 8). J Prosthet Dent 2005; 94 (1): 10-92.

4. Mishra R, Shvrayan A, Jain S, Mehta S. Fabrication of bar retained tooth supported mandibular overdenture. Int J Oral Health Sci 2014;4 (1): 37-41.

\section{How to cite this article:}

Sheen J. Arora et al.2017, Attachment Retained Overdenture. Int J Recent Sci Res. 8(3), pp. 16187-16189.

DOI: http://dx.doi.org/10.24327/ijrsr.2017.0803.0094 